\title{
Adherence with the standard treatment regimen for the treatment of COVID-19 patients in Bangabandhu Sheikh Mujib Medical University
}

\author{
Elora Sharmin, Md Sharfuddin Ahmed, Md Rasul Amin, Md Nazmul Hasan, Sayeda Papia, \\ Sheikh Foyez Ahmed, Rezwanul Quader
}

\begin{abstract}
Article Info
Department of Pharmacology, Bangabandhu Sheikh Mujib Medical University, Dhaka, Bangladesh (ES), Department of Community Ophthalmology, Bangabandhu Sheikh Mujib Medical University, Dhaka, Bangladesh (MSA), Department of cardiology, Bangabandhu Sheikh Mujib Medical University, Dhaka, Bangladesh (MRA, SFA), Department of Internal Medicine, Bangabandhu Sheikh Mujib Medical University (BSMMU), Dhaka, Bangladesh (MNH), Ashyian City Medical College, Dhaka, Bangladesh (SP), NICRH, Mohakhali, Dhaka (RQ).

For Correspondence:

Elora Sharmin

elora.sharmin@bsmmu.edu.bd

Received:

15 May, 2021

Accepted:

27 June, 2021

Available Online: $\quad 07$ July, 2021

ISSN: $2224-7750$ (Online) 2074-2908 (Print)

DOI: https://doi.org/10.3329/bsmmuj.v14i3.54675
\end{abstract}

Keywords: Corona virus disease 19 (COVID-19), standard treatment regime, Adherence to treatment.

Cite this article:

Sharmin E, Ahmed MS, Amin MR, Hasan MN, Papia S, Ahmed SF, Quader R. Adherence with the standard treatment regimen for the treatment of COVID-19 patients in Bangabandhu Sheikh Mujib Medical University. Bangabandhu Sheikh Mujib Med Univ J. 2021; 14 (COVID-19 Supplement): 13-18.

\section{Copyright:}

The copyright of this article is retained by the author(s) [Atribution CC-By 4.0]

Available at:

www.banglajol.info

A Journal of Bangabandhu Sheikh Mujib Medical University, Dhaka, Bangladesh

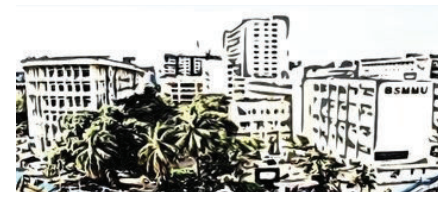

\section{Abstract}

The present study was conducted to evaluate the adherence to standard treatment regimen in Bangabandhu Sheikh Mujib Medical University (BSMMU) for the treatment of corona virus disease 19 (COVID-19) patients. The study assessed the awareness of prescribers, adherence to treatment guidelines and reasons for non-adherence. A questionnaire survey was conducted followed by a retrospective and prospective medical review and in-depth interview. The study revealed that $79.9 \%$ of key prescribers are aware of treatment guidelines. Adherence was $28.4 \%$ in moderate cases and $53.3 \%$ in severe cases and difference was highly statistically significant $(\mathrm{p}<0.001)$. Adherence in moderate cases was highest in patients with hypertension $(43.3 \%)$ and lowest in bronchial asthma $(16.6 \%)$. Adherence in severe cases was highest with diabetes (80\%) and lowest in bronchial asthma (20\%). In COVID-19 patients, the adherence was highest with International Guideline (96\%), followed by institutional Guideline (74.6\%) and National Guideline (23.5\%). There is significant variation in extent of adherence in different age group of patients, co-morbidities and categories of guidelines.

\section{Introduction}

The global pandemic of novel coronavirus disease 2019 (COVID-19) caused by severe acute respiratory syndrome coronavirus 2 (SARS-CoV-2) began in Wuhan, China, in December 2019. This novel Betacoronavirusis like severe acute respiratory syndrome coronavirus (SARS-CoV) and Middle East respiratory syndrome coronavirus (MERS$\mathrm{CoV})$; based on its genetic proximity, it likely originated from bat-derived coronaviruses with spread via an unknown intermediate mammal host to humans. ${ }^{1}$ The viral genome of SARS-CoV-2 was rapidly sequenced to enable diagnostic testing, epidemiologic tracking, and development of preventive and therapeutic strategies. Currently, there is no evidence from randomized clinical trials (RCTs) that any potential therapy improves outcomes inpatients with COVID-19. As coronavirus disease 2019 (COVID-19) spreads across the world as well as in our country, the health provide system of our community must prepare for the challenges associated with this pandemic. Streamlining of workflows for rapid diagnosis and isolation, clinical management, and infection prevention have played the vital role to combat the situation. The current World Health Organization (WHO) clinical management guidance document ${ }^{2}$ states "there is no current evidence to recommend any specific anti-COVID-19 treatment for patients with confirmed COVID-19". ${ }^{3}$ The guidance emphasizes the role of supportive care based on severity of illness, ranging from symptomatic treatment for mild disease to evidence-based ventilator management for ARDS and early recognition and treatment of bacterial infections and sepsis in critically ill patients. In this regard, the WHO recently announced treatments plan to launch a global "megatrial' (remdesivir, chloroquine or hydroxychloroquine, lopinavir/ritonavir, or lopinavir/ritonavir plus interferon- $\beta$ ) based on local drug availability. ${ }^{4}$

For the last 20 years' practice guidelines have become an increasingly popular tool for synthesis of clinical information and to change the clinical practices and improve quality of health care. ${ }^{5}$ Guideline can be developed nationally or locally. National guidelines are 
developed by professional bodies and working parties of special societies and the local or institutional guidelines are developed by the doctors of the institution who will use those. ${ }^{6}$ Like WHO recommended guidance in our country has a national guideline as well as an institutional guideline (BSMMU) for the treatment of COVID-19. As BSMMU is the only medical university in Bangladesh, its treatment practice should be monitored periodically to improve the quality of health care and to increase the awareness of the prescribers. In the developed countries, every medical institute follows either their national guideline or institutional guideline for the treatment of diseases. Bangabandhu Sheikh Mujib Medical University Hospital developed a COVID-19 treatment guideline. The effectiveness of such intervention principally depends on the acceptance of guideline by prescribers. This study has been performing to give special attention for the monitoring of the drug uses pattern and adherence with the existing guidelines of the different level prescriber of BSMMU COVID-19 unit. The assessment of adherence with the guidelines is important for clinical, educational, and economic purposes. ${ }^{7}$ Prescribing patterns need to be evaluated periodically to increase the therapeutic efficacy, decrease adverse effects, and provide feedback to prescribers. Drug utilization reviews are useful for obtaining information about drug use patterns.

\section{Materials and Methods}

This is an observational cross-sectional type of study, and the study was conducted among the COVID-19 patients who were admitted in COVID unit of BSMMU which maintained by the departments of Internal Medicine along with the physicians of all departments. This study was done under the supervision of the department of Pharmacology, Bangabandhu Sheikh Mujib Medical University (BSMMU). Initially a questionnaire survey was conducted among the key prescribers of the BSMMU hospital. The retrospective prescribing data was collected from the Record room of the BSMMU. The In-depth interview was conducted among 30 key prescribers of the different departments involve in the management of hospitalized COVID-19 patients and 285 COVID -19 positive patients who were admitted in BSMMU and among them 150 patient's data were collected from record room and 135 patient's data collected from the ward. Total duration of the study was from November 2020 to April 2021. Ethical issues of this study were reviewed and approved by Institutional Review Board of Bangabandhu Sheikh Mujib Medical University. Treatment records of individual cases containing clinical diagnosis with severity that were moderate and severe cases of SARS-CoV-2 diseases were considered as sample and samples were collected by consecutive sampling method. Treatment sheet containing moderate and severe COVID-19 cases, documented as discharged on request, absconded, and expired are excluded from this study. Sixty treatment sheets (WHO, 1993) of each condition moderate and severe was included and accordingly the sample size was 285 .

\section{Data collection}

Initially a questionnaire survey was conducted among the key prescribers (Chairman, Professor, Associate professor and Assistant professor) of different departments of BSMMU who were involve in the management of hospitalized COVID-19 patients and using a questionnaire that was a modified form of the previously developed one. ${ }^{8}$ (The purpose of the survey was to know about the preferred guidelines of the prescribers for COVID-19 they treat. Then a retrospective study was conducted by evaluating the treatment sheets conserved in the record room. The included cases were reviewed to evaluate the adherence with the treatment guidelines as mentioned by the key prescribers. Finally in-depth interview was conducted with the prescribers in order to assess their perception regarding treatment guidelines and reasons for nonadherence or less adherence with the guidelines. ${ }^{9-11}$ The trade name of the medicine commonly prescribed in the COVID-19 was checked and memorized by the researcher using Bangladesh National Formulary and the website of the Directorate General of Drug Administration. ${ }^{12}$ Data collection started from the most recently registered inpatient records to gradually backward manner. The treatment records of the COVID-19 patients where register was initially identified. Then the treatment sheets were reviewed for the inclusion and exclusion criteria to be included in the study. After inclusion, the treatment record of the COVID-19 patients was reviewed. Initially an ID was provided at top of the front page of the treatment sheet were confirmed moderate and severe COVID-19 cases and patient profile were mentioned. The following information were documented in review form: serial number of the prescription, date of the prescription, name of medicines in generic name (whether prescribed as generic or in trade name), formulation of medicines (either tablet or capsule or injections or inhaler or syrup or suspension or suppository), dose of the medicines, frequency of therapy, duration of hospital stays, route of medicine administration and number of medicines per prescription. The change of antimicrobial was evaluated to identify whether that was done on the basis of clinical findings or on laboratory findings (in follow up note). If that was based on laboratory findings, the prescription was considered adhered. In the prescription where the change was based on clinical findings, if the changed medicine was from the Guideline, the treatment was evaluated accordingly. Finally, after inclusion of the cases, information regarding confirmed moderate and severe COVID-19 cases, patient profile, records of prescribing and detail information of medicine was documented in an audit form. The treatment provided for the moderate and severe COVID-19 cases was only considered for the assessment. The remaining part of the prescription was not studied in this study.

\section{Assessment of the adherence}

Among the hospitalized COVID-19 patient's with of moderate and severe COVID-19 cases in adults, adherence was evaluated by matching with the National, international and institutional guideline moderate and severe cases of and consensus protocol of the key prescribers of the different departments .Prescriptions of SAR-CoV-2 were assessed for their adherence 
status by comparing them with the preferred guideline as mentioned by the key prescribers treating the disease. The clinical decisions were considered correct, only the following pharmacological issues mentioned in the guideline were compared: Selection of medicine, dose of medicine, frequency of therapy and route of administration of medicine.

\section{Working principle of adherence assessment}

If any prescription contains selected medicine as mentioned in the treatment guideline plus medicine unmentioned in the guideline, that prescription was not considered as 'adherence' because this study was conducted only to explore pharmacological issues, not the clinical judgment. In case of antibiotic, if the selection was according to guideline, only then it was considered as 'adherence' and further studied for dose, route and frequency. If any unmentioned antibiotic was prescribed, that was considered as 'non-adherence'. Guideline that has incomplete information about dose, route and frequency, recommendation of national formulary was considered. Initially, the name of the prescribed medicine was compared with the preferred guideline, whether that is mentioned or not in the Guideline. If the medicine was not from the mentioned list, then the prescription was considered as 'non-adherence' and the remaining steps to study dose, route and frequency were not performed. If the medicine was selected from the mentioned list, then the remaining steps, i.e., whether the mentioned dose, route and frequency were according to the recommendations of the guideline or not, was evaluated. Afterwards, the prescription was scored in the following manner: For every correct criterion, the prescription received one point and if the total score of the prescription was four, they were labeled as complete adherence. If any medicine that is mentioned in the treatment guideline as discouraged to prescribe or some clinical criteria were mentioned as prerequisite, in that case if prerequisite were not met, it was considered as 'non-adherence'. Adherence status was evaluated by a spreadsheet developed in Microsoft Office Excel by providing preset formula.

Analysis: Appropriate statistical test ( $Z$ test of proportion ) was done in this study for drawing an effective conclusion. Statistical analysis was done with the help of online statistical analysis calculator and Microsoft Office Excel.

\section{Results}

The initial questionnaire survey revealed that among the key prescriber requested to participate, 36.5\% (36/126) participated in the survey. Of the participated prescriber, $60.9 \%(30 / 36)$ of the key prescribers of BSMMU hospital are aware of treatment guidelines. Key prescribers from different departments who were involved in the management of hospitalized COVID-19 patients. Among the key prescriber 30 of them have mentioned the clinical conditions of SARSCoV-2 treated by them in admitted patients, of which International Guidelines (96\%), National Guideline $(23.5 \%)$, institutional guideline $(74.6 \%)$ and textbooks/journal/own experience $(13 \%)$ are followed as their guiding principles.

Amongst, 165 treatment sheets of moderate case ,47 completely adhered to the treatment guidelines. The adherence was highest in case of moderate case with HTN $43.3 \%$ and lowest in moderate case with bronchial asthma 16.6 $\%$. In case severe case with diabetes is highest $80 \%$ and lowest in bronchial Asthma .It was found that out of 285 treatment sheets 111 was completely adhered to the treatment guidelines in both moderate and severe case (table I).

Table-I

Appropriateness of selection of medicine and the adherence status with guidelines in adults COVID-19 moderate and severe treated in the corresponding departments

\begin{tabular}{|c|c|c|c|c|c|c|c|c|c|c|}
\hline \multirow[t]{2}{*}{ Case } & \multirow{2}{*}{$\begin{array}{l}\text { Number } \\
\text { of cases }\end{array}$} & \multicolumn{9}{|c|}{ Adhered } \\
\hline & & $\begin{array}{l}\text { Medicine } \\
\text { election }\end{array}$ & $\begin{array}{l}\text { Dose, route } \\
\& \text { frequency }\end{array}$ & Complete & $\begin{array}{c}\text { Dose } \\
\& \text { route }\end{array}$ & $\begin{array}{c}\text { Dose \& } \\
\text { frequency }\end{array}$ & $\begin{array}{c}\text { Route \& } \\
\text { frequency }\end{array}$ & Dose & Route & Frequency \\
\hline $\begin{array}{l}\text { Moderate case with } \\
\text { Bronchial Asthma }\end{array}$ & 30 & $11 / 30$ & $5 / 11$ & $5 / 30$ & $1 / 11$ & $5 / 11$ & $2 / 11$ & $7 / 11$ & $5 / 11$ & $3 / 11$ \\
\hline COPD & 30 & $28 / 30$ & $10 / 28$ & $8 / 30$ & $2 / 28$ & $10 / 28$ & $5 / 28$ & $10 / 28$ & $5 / 28$ & $2 / 28$ \\
\hline $\mathrm{DM}$ & 45 & $35 / 45$ & $5 / 35$ & $8 / 45$ & $2 / 35$ & $2 / 35$ & $18 / 35$ & $15 / 35$ & $5 / 35$ & $3 / 35$ \\
\hline HTN & 60 & $54 / 60$ & $32 / 54$ & $26 / 60$ & $5 / 54$ & $10 / 54$ & $25 / 54$ & $15 / 54$ & $38 / 54$ & $7 / 54$ \\
\hline Total & 165 & $128 / 165$ & $54 / 128$ & $47 / 165$ & $10 / 128$ & $27 / 128$ & $50 / 128$ & $47 / 128$ & $53 / 128$ & $15 / 128$ \\
\hline $\begin{array}{l}\text { Severe case with } \\
\text { Bronchial asthma }\end{array}$ & 15 & $8 / 15$ & $2 / 8$ & $3 / 15$ & $4 / 8$ & $5 / 8$ & $1 / 8$ & $2 / 8$ & $0 / 8$ & $1 / 8$ \\
\hline COPD & 25 & $20 / 25$ & $15 / 20$ & $8 / 25$ & $12 / 20$ & $1 / 25$ & $6 / 25$ & $5 / 25$ & $3 / 25$ & $1 / 25$ \\
\hline DM & 35 & $26 / 35$ & $10 / 26$ & $28 / 35$ & $5 / 26$ & $1 / 26$ & $3 / 26$ & $2 / 26$ & $1 / 26$ & $1 / 26$ \\
\hline HTN & 45 & $39 / 45$ & $20 / 39$ & $25 / 45$ & $17 / 39$ & $8 / 39$ & $4 / 39$ & $3 / 39$ & $1 / 39$ & $4 / 39$ \\
\hline Total & 120 & $93 / 120$ & $47 / 93$ & $64 / 120$ & $38 / 93$ & $15 / 93$ & $14 / 93$ & $12 / 93$ & $5 / 93$ & $7 / 93$ \\
\hline
\end{tabular}


Table-II

Proportion of prescription that completely adhered with the preferred guidelines of the key prescribers

\begin{tabular}{|c|c|c|c|c|c|c|}
\hline \multirow[t]{2}{*}{ Disease } & \multirow[t]{2}{*}{$\mathrm{n}$} & \multicolumn{2}{|c|}{ Complete adherence } & \multirow{2}{*}{$\begin{array}{c}\text { Total complete } \\
\text { adherence rate (\%) }\end{array}$} & \multirow[t]{2}{*}{ Z } & \multirow[t]{2}{*}{$\mathrm{P}$} \\
\hline & & Number & Percent & & & \\
\hline Moderate case with & & & & 28.4 & 0.96 & 0.001 \\
\hline Bronchial & 30 & 5 & 16.6 & & & \\
\hline COPD & 30 & 8 & 26.6 & & & \\
\hline DM & 45 & 8 & 17.7 & & & \\
\hline $\mathrm{HTN}$ & 60 & 26 & 43.3 & & & \\
\hline Total & 165 & 47 & 28.4 & & & \\
\hline Severe case with & & & & & & \\
\hline Bronchial asthma & 15 & 3 & 20 & 53.3 & & \\
\hline COPD & 25 & 8 & 32 & & & \\
\hline $\mathrm{DM}$ & 35 & 28 & 80 & & & \\
\hline HTN & 45 & 25 & 55.5 & & & \\
\hline Total & 120 & & 53.3 & & & \\
\hline
\end{tabular}

In this study, the proportion of total adhered prescription was $28.4 \%$ in moderate case of COVID-19 and severe case which was $53.3 \%$ in treated in BSMMU. The difference between totally adhered prescriptions of moderate case and severe case in BSMMU hospital was highly significant $(p<0.001)$. The adherence score regarding selection of medicine, dose, route, and frequency of administration as well as prescribing of other discouraged medicine. In moderate case with bronchial asthma in 24 to 44 above age group, incorrect medicine selection was the principal deviation. Whereas, in 20-23 age group, incorrect dose was the main contributor for nonadherence (table-II)

When adherence was assessed in the treatment of moderate and severe case of COVID-19 hospitalized patients with the corresponding preferred guideline of different categories., 96 $\%$ adherence was observed with international Guideline, followed by $74.6 \%$ with institutional Guidelines (Consensus Protocol) and $23.5 \%$ with National Guideline.

From the in-depth interview, different managerial issues like improper distribution, communication gap between the management and prescribers as well as lack of persuasion from the administration were mentioned as reason of non-adherence. Some scientific

issues also mentioned like faulty guideline development process, not updated, too complicated to follow and difference in clinical scenario than that mentioned in the Guideline. Furthermore, some prescriber related aspects like inadequate motivation, reluctance of junior prescribers, time constraints and empirical preference to individual's experience were mentioned by the key prescriber as reason of non-adherence. Proper distribution, dissemination and availability of the guidelines were suggested by the key prescriber as measure to improve adherence. They also advised to update the guideline regularly and to incorporate recent laboratory data into the guideline. Orientation and motivational programs were also mentioned by the key prescriber as measure to improve awareness as well as adherence. Finally, they suggested some stringent legal provision to enforce better adherence.

\section{Discussion}

Appropriateness of selection of medicine and the adherence status with guidelines in the treatment of COVID-19 cases. Though clinical decisions are based on scientific medical knowledge, a large gap remains between our knowledge and practice. ${ }^{12}$ Despite efforts to improve guideline adherence, success was very little due to different barriers. ${ }^{14}$ The present study revealed that two third of the key prescribers of BSMMU hospital was aware about treatment guidelines, though that varied in different departments. The observed level of awareness is slightly higher than the American licensed pediatricians, which indicate similar learning attitude of the physicians working both in rich and poor countries. ${ }^{15}$ This study revealed poor adherence in moderate case of COVID-19 patients. Non-adherence was mainly due to incorrect dose calculation and use of unmentioned medicine. Of the treated diseases, the adherence was highest in moderate case with hypertension $43.3 \%$. The adherence was lowest $16.6 \%$ percent in moderate case with bronchial asthma, 
where the gold standard was the National Guideline. The Australian and American studies revealed high adherence in case of bronchial asthma, moreover the US investigators have identified negative influence of use of cough suppressants and salbutamol suspension. ${ }^{17-18}$ The poor adherence in the present study perhaps negatively influenced by prescribing antimicrobials without documented evidence of required criteria, inappropriate selection, prescribing medicine discouraged like salbutamol and antihistamine. COPD cases adhered with the National Guideline, which is because of prescribing antimicrobials in response to suspicion of pneumonia and bacterial coinfections without documented evidence.

The adherence score regarding selection of medicine, dose, route, and frequency of administration as well as prescribing of other discouraged medicine. In moderate case with pneumonia in 24 to 44 above age group, incorrect medicine selection was the principal deviation. Whereas, in 20-23 age group, incorrect dose was the main contributor for non-adherence. This is further worsened by the fact that recommended dosing of the National Guideline does not correspond with that of Bangladesh National Formulary. The present study revealed that in severe case patient's 53.3 percent of the prescription adhered with the guideline. The adherence was highest in DM, HTN and lowest in bronchial asthma. Highest adherence in diabetes and hypertension was perhaps because the most potential area of deviation, some medicines which were produce complication in this comorbidity the key prescribers can't prescribe due to financial considerations and other social issues. Moreover, the recommendations of guideline on selection of medicine were flexible as there were no medicine was mentioning clinical judgment of the prescribers, which provided adequate flexibility that contributed positively on adherence. Motivation and social awareness kept the prescribers away from the deviation. Adherence was low in bronchial asthma, which was due to antimicrobial and antihistamine prescribing without documented required criteria as well as use of theophylline in some cases. There was also some reluctance on the part of residents regarding dose calculation and frequency of administration. Though adherence was little higher in chronic obstructive pulmonary disease (COPD) than asthma, $32 \%$ in case of COPD, the influencing factors possibly were similar. Most of the patients adhered to the international and institutional guideline; the remaining non-adherence was due to little reluctance during rounding up of the calculated dose. Adherence rate is more in case of international guideline followed by institutional guideline, i.e., consensus protocol and lowest with National guideline. This feature reflects the better acceptance of international guideline by the prescribers of BSMMU than that of the institutional and national. As in this pandemic situation most of the drugs were getting emergency authorization. The present study revealed variation in adherence in severity of patients, which perhaps mainly because of difference in severity as well as presence of comorbidity, structure of guidelines and issue of individual dose calculation as well as availability of drug. Audit and feedback have been used as a strategy for improving clinical practice behaviors as well as cost effectiveness of the prescriptions. 19,20 Therefore, well structured 'audit and feedback' of the observations of the present study might have some positive impact on the adherence status. From the in-depth interview, the key prescribers have identified some managerial deficiencies like improper distribution, inadequate persuasion, and communication gap as the reasons for non-adherence. Prescriber also mentioned some scientific defects like faulty development procedure, too rigid to cope with the difference in clinical response and guidelines are also too complicated. In addition, the prescribers noticed some lack of motivation, reluctance on the side of young residents and time constraint to comply with the documentation requirements of the guideline. To improve, changes at different levels of medical education, particularly in teaching and evaluation system of pharmacology, might be effective. ${ }^{21}$ The present study revealed that though a high proportion of prescriber know about the guideline, but they follow the guideline to limited extent, which reiterated the gap between prescriber's knowledge and practice. ${ }^{22}$ The suggestions of the prescribers to improve adherence corresponds with the previous recommendations from Netherland. ${ }^{22-24,25}$

\section{Conclusion}

There is a significant variation in extent of adherence SARS$\mathrm{CoV}-2$ treatment in different by prescribers of BSMMU Hospital. The findings of the present study might be helpful for better designing of subsequent institutional and national treatment guidelines as well as enforcement of guidelines in other institutions of Bangladesh.

\section{Financial Support}

Bangabandhu Sheikh Mujib Medical University

\section{Conflict of Interest}

Authors declare no conflict of interest.

\section{References}

1. Cascella, M, Rajnik, M, Cuomo, A, Dulebohn, SC \& Di Napoli, R2020, 'Features, evaluation and treatment coronavirus (COVID-19)', in Statpearls [internet]. StatPearls Publishing. 
2. Worldometer. Coronavirus Cases. 2020. Available: https: //www.worldometers.info/coronavirus/coronavirus-cas es/. Accessed: 4 May 2020.

3. Huq, S \& Biswas, $\mathrm{R}$ no date, 'COVID-19 in Bangladesh: Data deficiency to delayed decision', jogh.org. Available at:http:/ / www.jogh.org/documents/issue202001/jogh-1 0-010342.pdf (Accessed: 4 May 2020).

4. Institute of Epidemiology, Disease Control and Research. General information COVID-19 2020. Available: https://www.iedcr.gov.bd/index.php/component/conte nt/article/73-ncov-2019. Accessed: 1 April 2020.

5. Borges do Nascimento IJ, Cacic N, Abdulazeem HM, von Groote TC, Jayarajah U, Weerasekara I, Esfahani MA, Civile VT, Marusic A, Jeroncic A, Carvas Junior N. Novel coronavirus infection (COVID-19) in humans: a scoping review and meta-analysis. Journal of clinical medicine. 2020 Apr;9(4):941.

6. Liu Y, Gayle AA, Wilder-Smith A, Rocklöv J. The reproductive number of COVID-19 is higher compared to SARS coronavirus. Journal of travel medicine. 2020 Mar 13.

7. Chen T, Wu DI, Chen H, Yan W, Yang D, Chen G, Ma K, Xu D, Yu H, Wang H, Wang T. Clinical characteristics of 113 deceased patients with coronavirus disease 2019: retrospective study. bmj. 2020 Mar 26;368.

8. Rahman MS, Siddika AN. Antimicrobial Guideline of BSMMU: Assessment of Compliance.

9. Haque MM. ABC of research methodology and biostatistics. Department of Biochemistry, BSMMU, Dhaka, 2009.

10. Islam MS, Rahman MS, Misbahuddin M. Impact of'Prescription Audit \& Feedback'on Pattern of Prophylactic Antimicrobials in Cesarean Section: a Cost Reduction Perspective. Bangladesh Journal of Physiology and Pharmacology. 2007:1-9.

11. Das AK, Rahman MS. Prescribing vitamins at primary health care level: Exploration of facts, factors and solution. Bangladesh Journal of Pharmacology | | | 2010;5(2):92-7

12. Director General of Health Services (DGHS). Health Bulletin, 2012. Directorate of Drug Administration (DDA). Bangladesh Medical Association (BMA), Bangladesh Pharmaceutical Society (BPS). BangladeshNational Formulary. 3rded. Dhaka, 2006.
13. Sackett DL, Rosenberg WM, Gray JM, Haynes RB, Richardson WS. Evidence based medicine. BMJ: British Medical Journal. 1996 Jul 20;313(7050):170.

14. Lugtenberg M, Burgers JS, Besters CF, Han D, Westert GP. Perceived barriers to guideline adherence: a survey among general practitioners. BMC family practice. 2011 Dec;12(1): 1-9.

15. Christakis DA, Rivara FP. Pediatricians' awareness of and attitudes about four clinical practice guidelines. Pediatrics. 1998 May 1;101(5):825-30.

16. Rahman MS. Changes required in pharmacotherapy teaching to ensure rational use of drugs. Bangladesh J Physiol Pharmacol. 1995;11(1):38-9.

17. Sanders TR, Roberts CL, Gilbert GL. Compliance with a protocol for intrapartum antibiotic prophylaxis against neonatal group B streptococcal sepsis in women with clinical risk factors. Infectious diseases in obstetrics and gynecology. 2002 Jan 1;10(4):223-9.

18. Rastogi D, Shetty A, Neugebauer R, Harijith A. National Heart, Lung, and Blood Institute guidelines and asthma management practices among inner-city pediatric primary care providers. Chest. 2006 Mar 1;129(3):619-23.

19. Hysong SJ, Best RG, Pugh JA. Audit and feedback and clinical practice guideline adherence: making feedback actionable. Implementation science. 2006 Dec;1(1):1-0.

20. Islam MS, Rahman MS, Misbahuddin M. Impact of' Prescription Audit \& Feedback'on Pattern of Prophylactic Antimicrobials in Cesarean Section: a Cost Reduction Perspective. Bangladesh Journal of Physiology and Pharmacology. 2007:1-9.

21. Rahman MS. Changes required in pharmacotherapy teaching to ensure rational use of drugs. Bangladesh J Physiol Pharmacol. 1995;11(1):38-9.

22. Sackett DL, Rosenberg WM, Gray JM, Haynes RB, Richardson WS. Evidence based medicine. BMJ: British Medical Journal. 1996 Jul 20;313(7050):170.

23. Lugtenberg M, Burgers JS, Besters CF, Han D, Westert GP. Perceived barriers to guideline adherence: a survey among general practitioners. BMC family practice. 2011 Dec; 12(1):1-9.

24. Mistiaen P, Bloemendal E, Weenink J, Harmsen M. Guideline adherence in the Netherlands: A systematic review, 2012;

25. Cohn JN. Monitoring adherence to guidelines. Circulation: Heart Failure 2008; 1: 8788. 